\title{
Iris Image Retrieval Based on Macro-features
}

\author{
Manisha Sam Sunder and Arun Ross \\ West Virginia University \\ manishasamsunder@gmail.com,arun.ross@mail.wvu.edu
}

\begin{abstract}
Most iris recognition systems use the global and local texture information of the iris in order to recognize individuals. In this work, we investigate the use of macro-features that are visible on the anterior surface of RGB images of the iris for matching and retrieval. These macro-features correspond to structures such as moles, freckles, nevi, melanoma, etc. and may not be present in all iris images. Given an image of a macrofeature, the goal is to determine if it can be used to successfully retrieve the associated iris from the database. To address this problem, we use features extracted by the Scale-Invariant Feature Transform (SIFT) to represent and match macro-features. Experiments using a subset of 770 distinct irides from the Miles Research Iris Database suggest the possibility of using macrofeatures for iris characterization and retrieval.
\end{abstract}

\section{Introduction}

The iris textural pattern has been used as a biometric cue for the purpose of human recognition. Most iris recognition systems exploit the global and local texture information of the iris in order to characterize its pattern. They do not consider specific anatomical features present on the surface of the iris. In recent literature $[11,1,8]$, local features or keypoints representing intensity discontinuities in the spatial domain have been used for iris recognition. However, these methods do not explicitly extract features corresponding to macrofeatures such as moles, freckles, nevi or melanoma, and other characteristics such as sectoral heterochromia ${ }^{1}$. In this work, we investigate the potential of using macrofeatures (see Figure 1) for iris matching and retrieval. The macro-features of the iris, as defined in this work

\footnotetext{
${ }^{1}$ The definition of macro features can be expanded to include other structures such as Wolfflin spots, Brushfield spots (which are more evident in NIR images) and Lisch nodules. In this work, crypts are not considered as macro-features.
}

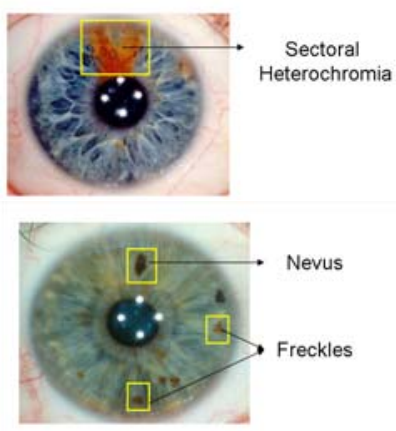

Figure 1: Examples of macro-features in the Miles Database.

correspond to pigmentation details ${ }^{2}$ and begin to form in childhood. Melanomas are uncontrolled reproduction of melanocytic pigment granules, and melanomas in the eye can be intraocular or extraocular. Intraocular melanomas are melanomas in the uveal tract consisting of the iris, ciliary body and choroid. The average age of the people diagnosed with iris melanoma is 40 and it is very rare $[9,2]$. The melanomas may change in size and shape with time, and usually distort the iris stroma and pupil [4]. Iris melanoma (cancerous tumors that increase in size with time), freckles, nevi (benign tumors that remain constant in size), moles and sectoral heterochromia (melanoma over a sector of the eye 1) are the structures considered in this work. The macro-features are usually yellow, tan, and brown in color. Latanoprost treatment may cause changes in iris color but typically does not cause changes in nevi or freckles with respect to their shape, color and texture [10]. Although these structures may not be permanent, the goal of this paper is to introduce the possibility of using these structures for the purpose of image retrieval. The observation that these features may not be present in every iris makes them useful for iris image retrieval from a large database. These features are prominently observed in blue and light-colored irides and are less prominent or

\footnotetext{
${ }^{2}$ So they are associated with RGB color images of the iris (rather than their infrared counterparts). In this work, the RGB images are converted to gray scale for further processing.
} 
rare in dark-colored iris [2]. They can be viewed as a soft biometric trait.

Recent works in face recognition have investigated the use of moles [6], scars and freckles for the purpose of image recognition and retrieval [3]. Such features are also useful in the field of forensics [7]. However, in the case of the iris biometric, the use of local anatomical oddities for comparing irides has not been studied. Such an approach has several benefits: (a) The use of macro-features would represent an alternate way to perform iris recognition in the RGB domain; (b) Forensic experts can use these macro-features to visually compare two irides by using "point-to-point" matching; (c) These features, when available (and visible), can be helpful in the recognition of partial irides.

The purpose of this work is to demonstrate the possibility of using images of macro-features to retrieve pertinent irides from a database of iris images. The following sections discuss the approach used to meet this goal.

\section{Macro-feature characterization}

Since macro-features can take on arbitrary shapes, it is difficult to characterize them using parametric models. Furthermore, depending upon the pigmentation of the iris, the structure of these macro-features may not be pronounced. Thus, in this work we employ the Scale Invariant Feature Transform (SIFT) [5] to characterize the macro-features and use the ensuing template to retrieve pertinent irides from a database. The SIFT technique associates keypoints with an image.

\subsection{Keypoint detection and representation}

Keypoints correspond to local features in the image representing color or intensity discontinuities. Interest point detectors may be used to identify keypoints for the purpose of object recognition, image retrieval, tracking and other image matching tasks. The scale Invariant Feature Transform (SIFT) [5] based on DoG detector can be used to detect keypoints that are invariant to changes in scale and orientation. These points are stable at multiple scales and are not affected by the change in view angle The scale space image, $L(x, y, \sigma)$ is constructed from difference of images convolved using the variable-scale Gaussian, $G(x, y, \sigma)$, where, $\sigma$ defines the scale and $I(x, y)$ is the input image. The difference of Gaussians between two scales which are separated by a multiplicative factor $k$ is defined as, $D o G=(G(x, y, k \sigma)-G(x, y, \sigma)) * I(x, y)$.

In order to detect the keypoints in an image, the input image is incrementally convolved with Gaussians to produce a stack of images separated by a factor of $k$ in scale. The Difference of Gaussian (DoG) images are obtained by subtracting images of adjacent scales. Extrema points corresponding to image discontinuities and representing maximum or minimum value among 8 neighbors in the current image and 9 neighbors each in the scales above and below the current DoG image are extracted. These extrema points are further processed to remove unstable extrema points with low contrast and instability [5], resulting in the final set of keypoints for an image.

Each keypoint is associated with a descriptor or feature vector. The feature vector is extracted based on the orientation of the keypoint. The orientation of the keypoint is obtained using the Gaussian smoothed image $L$ from which it is detected. A 36 bin orientation histogram covering the $[0,360]$ interval is computed around the keypoint using the gradient magnitude $m(x, y)$ and orientation $\theta(x, y)$ at each location of the image where $m(x, y)=[(L(x+1, y)-L(x-$ $\left.\left.1, y))^{2}+(L(x, y+1)-L(x, y-1))^{2}\right)\right]^{\frac{1}{2}}$, and $\theta(x, y)=$ $\tan ^{-1}\left(\frac{(L(x, y+1)-L(x, y-1))}{(L(x+1, y)-L(x-1, y))}\right)$. The orientation of the keypoint is computed as the highest peak in the orientation histogram associated with it. The feature vector is obtained by sampling the gradient magnitude and orientations within a descriptor window of size 16x16 around the keypoint as explained in [5]. The keypoint descriptor is computed as an 8 bin orientation histogram over $4 \times 4$ sample regions within the descriptor window, resulting in a descriptor of dimension $4 \times 4 \times 8$.

Figure 2 shows examples of some of the macrofeatures used in the experiment for image retrieval and the SIFT keypoints detected on a few macro-features. Note that multiple keypoints can be computed for a single macro-feature. Figure 3 shows the SIFT keypoints detected on two iris image; here, the direction of the arrow is the orientation assigned to the keypoint detected.

\subsection{Matching}

Matching is the process of comparing the keypoints of the input macro-feature against the set of keypoints extracted from a gallery iris image. The output of the matching algorithm is the number of keypoint correspondences obtained between the query macro-feature and the iris image in the database. Each query keypoint is matched with all the keypoints of the gallery iris image and a correspondence is obtained by comparing the top two closest matches for the query keypoint. The cosine similarity measure, $\theta$, is used to estimate the similarity between two 128-dimensional keypoint descriptors $\operatorname{des}_{1}$ and $\operatorname{des}_{2}$. The angle, $\theta$, is defined as, $\theta=\cos ^{-1}\left(\frac{\operatorname{des}_{1}^{T} \operatorname{des}_{2}}{\left\|\operatorname{des}_{1}\right\|\left\|\operatorname{des}_{2}\right\|}\right)$. 

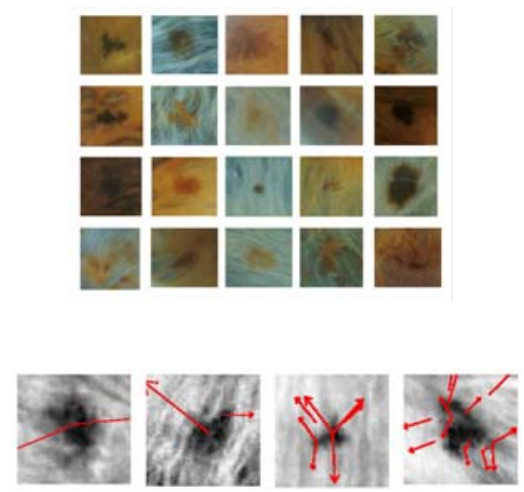

Figure 2: Examples of macro-features (geometrically normalized for display purposes) and detection of keypoints on macro-features.

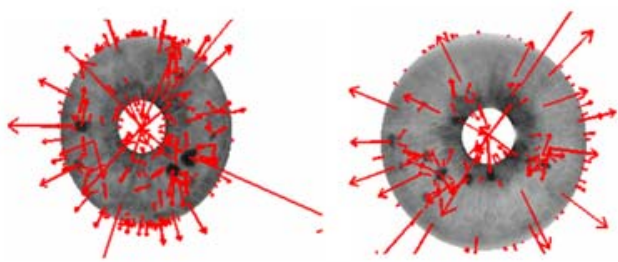

Figure 3: Keypoint detection on sample iris images.

A correspondence between a macro-feature keypoint and an iris image keypoint is established if the $\theta$ value associated with this match is lesser than 0.6 times the $\theta$ value associated with the next closest match. Figure 4 shows the results of matching keypoints extracted from the query macro-feature with that of an iris image in the gallery. It shows that SIFT features are reliable for matching even when the macro-feature or the iris image is rotated with respect to the macro-feature.
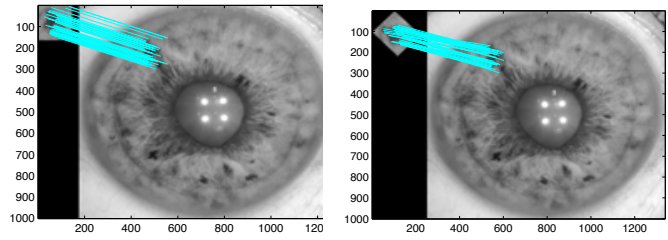

Figure 4: Example of matching a macro-feature with an iris image (Left) and a macrofeature rotated by $45 \mathrm{de}$ grees with an iris image (Right).

\section{Database}

The Miles Research ${ }^{3}$ database consists of high resolution iris images in the standard RGB domain. Images are captured using a Nikon digital SLR and a coaxial biometric illuminator producing images with high resolution and uniform illumination. The database consists of 770 gallery images of which 390 images contain 1,187 macro-features (manually labeled). The probe images consisting of the macro-features are extracted from the gallery images in this database. The database is pre-sorted from blue to brown irides. Figure 5 shows the distribution of macro-features across the database. As mentioned earlier, the distribution suggests that the brown irides have fewer macro-features compared to light colored irides.

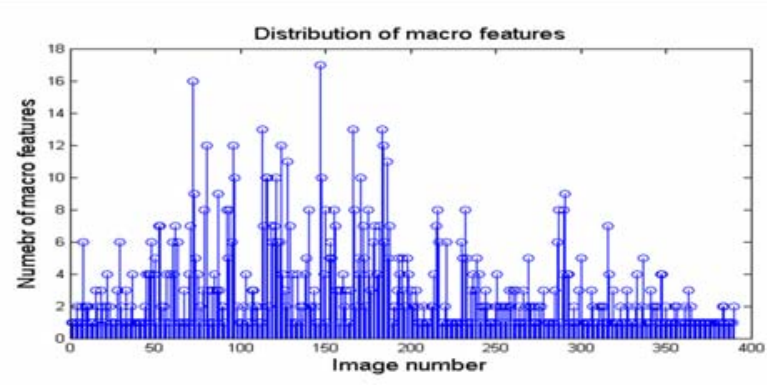

Figure 5: Distribution of macro-features across the pre-sorted database.

\section{Experimental results}

Based on the color of the iris, the red, green or blue component of the multichannel iris image is used to extract the local key points for both the macro-feature and the gallery iris image. The query macro-feature is subjected to SIFT keypoint detection and characterized using the resultant SIFT descriptors. The query keypoints $Q$ of an input probe macro-feature are matched against the keypoints of each gallery image $G_{i}, i=1,2, \ldots n$, and the top $K$ identities are retrieved based on the number of keypoint correspondences between them. Two experiments are conducted to evaluate the performance of image retrieval using macro-features. The first experiment uses the keypoints of one macro-feature of an iris image to retrieve the top $K$ iris images from the gallery database, while the second experiment uses keypoints from all the macro-features of an iris image to retrieve the top $K$ iris images from the gallery database.

Evaluation is performed by determining the number of times the true iris image containing the probe macrofeature is retrieved from the gallery space. A hit at rank $k$ indicates that the iris image containing the probe macro-feature is correctly retrieved as one among the top $k$ images. The first experiment is performed using all the 1,187 macro-features as probe images and all the

\footnotetext{
${ }^{3}$ http://www.milesresearch.com/
} 
770 irides as gallery images. A hit-rate of $87.02 \%$ and $88.2 \%$ is observed at ranks 1 and 2 , respectively. In the second experiment, all the macro-features pertaining to an iris image are considered to be a single probe; thus, a total of 390 probes are available in this experiment. The gallery image is once again composed of all 770 irides. A hit-rate of $92.8 \%$ at rank 1 is observed for this database of 770 iris images where a subset of 380 images do not have any macro-features.

Errors in the retrieval process could be attributed to the detection of very few keypoints (sometimes zero) around a macro-feature (either in the probe or in the gallery). For example, in experiment 1 , it was observed that the miss rate of $\sim 12.97 \%$ at rank-1 was actually caused by $2.6 \%, 5.8 \%, 3.3 \%$ and $1.27 \%$ of the probe macro-features that had zero, one, two and three or more SIFT keypoints, respectively. Fewer keypoint detections could occur due to the very small size and uniformly smooth texture of a macro-feature. This results in the inaccurate matching of the macro-feature against that of the gallery iris image. In order to understand the resilience of the retrieval scheme to noise, the two experiments were repeated after synthetically corrupting the probe macrofeatures with Poisson noise. Experiments 1 and 2 resulted in rank-1 hit rates of $78.01 \%$ and $87.69 \%$, respectively. However, the experiments do indicate that macro-features may be used as a soft biometric trait for iris image retrieval and, perhaps, can be used in conjunction with existing iris encoding algorithms for improving recognition accuracy in the RGB domain.

\section{Summary and Future Work}

The paper proposes the use of macro-features for the purpose of iris image retrieval. Although these features have a low frequency of occurence, preliminary experiments suggest that these structures may be used in addition to traditional features for the purpose of iris retrieval. Thus, they serve as a soft biometric trait. These features may also be used for point-to-point matching of iris images. The use of the SIFT method is also advantageous as it replaces the actual iris image in the database with the keypoints detected on it.

The inability of the proposed approach to retrieve the correct iris images in some instances provides an opportunity for further improvement. The use of advanced matching techniques based on texture, color and location of macro-features is being currently studied. In addition, experiments involving macro-features that change in size - such as melanoma and sectoral heterochromia - need to be conducted for the purpose of understanding their utility in databases containing timelapsed iris images. Also, a method to automate macrofeature extraction is being investigated.

\section{Acknowledgements}

This work was funded in part by the Center for Identification Technology Research (CITeR) at WVU. Thanks to Jon Miles from Miles Research for granting us access to the database used in this work.

\section{References}

[1] C. Belcher and Y. Du. Region-based sift approach to iris recognition. Optics and Lasers in Engineering, 47:139$147,2009$.

[2] E. Henderson and E. C. Margo. Iris melanoma. Archives of Pathology and Laboratory Medicine, 132(2):268272, February 2008.

[3] A. K. Jain and U. Park. Facial marks: Soft biometric for face recognition. In IEEE International Conference on Image Processing (ICIP), November 2009.

[4] A. D. Lee and J. E. Higginbotham. Clinical Guide to Comprehensive Ophthalmology. Thieme Medical Publishers, 1 edition, 1999.

[5] D. Lowe. Distinctive image features from scale invariant keypoints. International Journal of Computer $\mathrm{Vi}$ sion, 60(2):91-110, November 2004.

[6] J. S. Pierrard and T. Vetter. Skin detail analysis for face recognition. In Computer Vision and Pattern Recognition (CVPR), pages 1-8, 2007.

[7] N. Spaun. Forensic biometrics from images and video at the federal bureau of investigation. In Biometrics: Theory, Applications and Systems (BTAS), September 2007.

[8] H. Sung, J. Lim, J. Park, and Y. Lee. Iris recognition using collarette boundary localization. In International Conference on Pattern Recognition, volume 4 of 23-26, pages 857-860, August 2004.

[9] A. Tantri, W. Alward, and T. Weingeist. Iris melanoma: 47 yo man referred in 1997 for evaluation of iris lesion os. Technical report, EyeRounds.org, February 2005.

[10] P. Wistrand, J. Stjernschantz, and K. Olsson. The incidence and time-course of latanoprost-induces iridial pigmentation as a function of eye color. Survey of Ophthalmology, 41(2):129-138, February 1997.

[11] W. Yang, L. Yu, L. Guangming, and K. Wang. Iris recognition based on location of key points. In ICBA, July 2004. 